\title{
Retracted: Positive Effects against UV-A Induced Damage and Oxidative Stress on an In Vitro Cell Model Using a Hyaluronic Acid Based Formulation Containing Amino Acids, Vitamins, and Minerals
}

\author{
BioMed Research International \\ Received 28 November 2020; Accepted 28 November 2020; Published 26 February 2021 \\ Copyright (c) 2021 BioMed Research International. This is an open access article distributed under the Creative Commons \\ Attribution License, which permits unrestricted use, distribution, and reproduction in any medium, provided the original work \\ is properly cited.
}

BioMed Research International has retracted the article titled "Positive Effects against UV-A Induced Damage and Oxidative Stress on an In Vitro Cell Model Using a Hyaluronic Acid Based Formulation Containing Amino Acids, Vitamins, and Minerals" [1]. As raised on PubPeer [2], there are duplicated figure panels within Figure 2(a):

(i) The CTR $(16 \mathrm{~h})$ and CTR $(32 \mathrm{~h})$ panels appear to be identical

(ii) The M-HA (48h) and M-HA (96h) appear to be identical

(iii) Skinkò E (48h) and Skinkò E (96h) appear to be identical

(iv) Skinkò E (72h) and CTR (96h) appear to be identical

(v) The same cluster of cells can be seen in Skinkò $\mathrm{E}$ (48 h), Skinkò E (72 h), and Skinkò E (96h).

The authors said that the duplications between M-HA (48 h) and M-HA (96h) and CTR (96) and Skinkò E (72 h) were mistakes introduced during manuscript preparation. A satisfactory explanation was not provided for the remaining concerns and the article is therefore being retracted with the agreement of the editorial board. The authors do not agree to retraction.

\section{References}

[1] A. Stellavato, A. V. A. Pirozzi, S. Donato et al., "Positive Effects against UV-A Induced Damage and Oxidative Stress on an In Vitro Cell Model Using a Hyaluronic Acid Based Formulation Containing Amino Acids, Vitamins, and Minerals," BioMed Research International, vol. 2018, Article ID 8481243, 11 pages, 2018.

[2] “PubPeer," 2019, https://pubpeer.com/publications/ EE4777EBD82DF143A1A9E791999AD1. 